\title{
A Conceptual Architecture and a Framework for Dealing with Variability in Mulsemedia Systems
}

\author{
Estêvão Bissoli Saleme \\ Federal University of Espírito Santo \\ Vitória, ES, Brazil \\ estevaobissoli@gmail.com
}

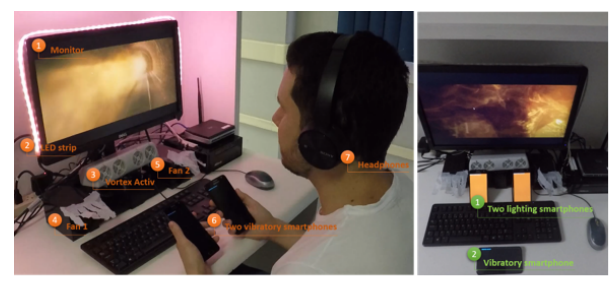

(a)

\author{
Celso A. S. Santos \\ Federal University of Espírito Santo \\ Vitória, ES, Brazil \\ saibel@inf.ufes.br
}

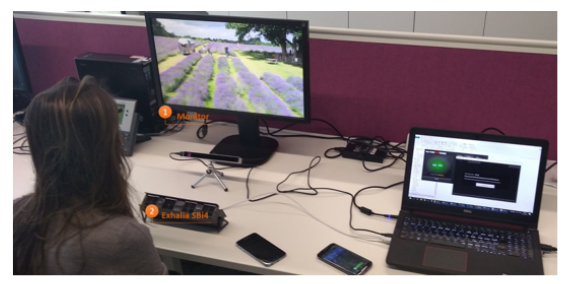

(b)

\author{
Gheorghita Ghinea \\ Brunel University London \\ London, UK \\ george.ghinea@brunel.ac.uk
}

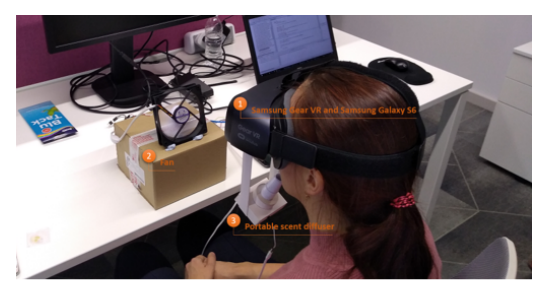

(c)

Figure 1: Different scenarios using the framework: (a) Video Clip Enriched with External Light, Smell, Vibration, and Wind, (b) Smell-intensive System, and (c) $360^{\circ}$ VR Mulsemedia System [20].

\begin{abstract}
The candidate's thesis is within the emerging topic of mulsemedia systems, which encompass audiovisual content associated with multisensory effects, users' quality of experience, and human-computer interaction. It presents a conceptual architecture and a framework that take into account the challenges and requirements for mulsemedia delivery systems identified from the gaps and shortcomings in related work. Furthermore, the work's outcome brings multiple and valuable contributions relying on a complex and solid experimental setup, which have been shared and published in relevant journals and conferences.
\end{abstract}

\section{KEYWORDS}

mulsemedia systems, multimedia applications, variability, conceptual architecture, software integration, frameworks

\section{INTRODUCTION}

This paper presents a summary of the results obtained in the first author's PhD thesis defended in the Postgraduate Program in Computer Science in 03/12/2019. The work was developed by the author, in a period of 37 months, under the supervision of the remaining authors.

The increasing interest in digital immersive experiences has drawn the attention of researchers into understanding human perception whilst adding sensory effects to multimedia systems such as Virtual Reality (VR) and Augmented Reality (AR) applications, content players, and games. These so-called mulsemedia-multiple sensorial media-systems [16, 17] are capable of delivering wind, smell, vibration, among others, along with audiovisual content to

In: II Concurso de Teses e Dissertações (CTD 2020), São Luís, Brasil. Anais Estendidos do Simpósio Brasileiro de Sistemas Multimídia e Web (WebMedia). Porto Alegre: Sociedade Brasileira de Computação, 2020.

(C) 2020 SBC - Sociedade Brasileira de Computação.

ISSN 2596-1683 enhance users' Quality of Experience (QoE) and to improve immersiveness in applied areas such as entertainment, healthcare, education, culture, and marketing.

There have been identified incipient proposals of conceptual architectures and frameworks inextricably restricted to MPEG-V, IoT approaches applied to mulsemedia, and SDKs and APIs to interface with specific sensory effects devices, such as wind fans, scent emitters, and vibration chairs. These devices, in turn, are constantly evolving, making it difficult to update applications to be compatible with them [14]. A plethora of issues have hindered the development of models and tools that takes variability of scenarios of usage into account as reviewed in related work. Notably, high coupling of software, lack of support for varied protocols to interact with applications and devices, absence of solutions able to work with timeline- and event-based multimedia applications, unavailability of mechanisms to support more than one SEM standard, scarcity of means to compensate delays introduced for some hardware or connectivity and communication protocols, hardware heterogeneity tackled isolated by some SDKs or APIs just in haptic, lack of schemes to adapt solutions to different profiles of usage without changing their code, and code reliance strictly on existent technologies, protocols, and standards without caring for future extensibility [20]. Every time a mulsemedia application is needed, new software is built mostly from scratch. This process has proven to be demanding, time-consuming, and costly mainly because it requires researchers and developers alike to gain knowledge about new devices, connectivity, communication protocols, and other particulars. The fact is that building such systems imposes several challenges and requirements (discussed in the thesis) due mainly to their ever-evolving and heterogeneous traits [19]. As a result, few mulsemedia systems have remained reusable to be applied to different research purposes as opposed to the use of open mulsemedia datasets [20]. 
Therefore, the thesis [11] introduces a decoupled conceptual architecture to deal with variability of scenarios in mulsemedia delivery systems filling those gaps and shortcomings, which includes recommendations to cope with the variation of end-user applications and sensory effect devices through the support and reuse of even unforeseen communication and connectivity protocols, and Sensory Effects Metadata (SEM). The conceptual architecture is grounded on the separation of concerns by dividing a mulsemedia system into five main layers: (1) Mulsemedia Applications, which isolates multimedia issues; (2) Communication Broker, which provides the reuse of mulsemedia services provided by sensory effect renderers through different communication protocols; (3) Sensory Effects Processing, which copes with metadata processing regardless of the SEM standard; (4) Connectivity Layer, which allows the reuse of different connectivity protocols for sensory effect devices independently of implementation of commands for devices; and (5) Sensory Effects Rendering, to represent devices that deliver sensory effects to end-users.

To evaluate it, an open-source and robust mulsemedia framework was developed. It allows researchers and developers to either undertake mulsemedia experiments or to take it as a reference to follow the conceptual architecture. The framework supports multicommunication and multi-connectivity protocols, multi-standards, and allows the accommodation of new technology relying on its set of architectural and design patterns to be applied successfully. It provides services to mulsemedia applications through a transparent communication broker and is able to work with timeline- and event-based applications. Moreover, the framework presents a set of configurable parameters to customize the solution according to the needs imposed by mulsemedia applications, offers a mechanism to calibrate delay for each component it works with, and provides a debug and flexible simulation scheme [20]. Then, a performance assessment was carried out on communication protocols for the integration between event-based applications, whereby temporal restrictions play a role, and the framework. Results indicated statistically significant differences in response time providing directions for optimized integrations. Finally, a user QoE subjective evaluation comparing a monolithic mulsemedia system with this framework was undertaken with results suggesting no evinced statistically significant differences in user-perceived QoE between the systems under different aspects. Therefore, it is hoped that the work fosters the area of Multimedia and Human-Computer Interaction (HCI) in the sense that researchers can leverage either the conceptual architecture to design mulsemedia delivery systems or the framework to carry out their experiments.

\section{EVALUATIONS}

In order to evaluate the candidate's thesis's proposal, case studies, performance, and subject experiments were undertaken and the results are briefly introduced next.

\subsection{Case Studies on Variability}

The case studies carried out present heterogeneous real-world scenarios of usage in which the conceptual architecture was materialized into a framework to empirically validate it [20]. These studies include different profiles of usage successfully undertaken to adjust the framework for (a) a mulsemedia environment comprised of video clips enriched with external light, smell, vibration, and wind delivered a system composed of a media player compatible with MPEG-V, DIY devices, smartphones, and a commercial scent device; (b) a scenario of usage whereby a proprietary device is handled by the framework to deliver different intensities of smell; and (c) a $360^{\circ}$ VR mulsemedia system composed of an HMD display with a portable scent diffuser attached to it and a wind device to engage users in a $360^{\circ}$ environment augmented with smell and airflow. Figure 1 portrays these different environments using the same framework with distinct configurations. The results showed the framework's capability to adapt itself to different scenarios of usage through configuration, without requiring code change. These scenarios turned into a setting for other studies $[1,4,9,19]$.

\subsection{Performance Assessment}

This study made a comparison between different protocols (CoAP, MQTT, UPnP, and WebSocket) that convey information between mulsemedia applications to the implemented framework. Four mock applications were created for each one. The interaction between the two sides is given by two steps: (1) a loop sequence to send SEM from the mulsemedia application to the framework; and (2) a real-time communication to start rendering sensory effects after an occurrence of an event in the mulsemedia former. This communication strategy was an improvement in the face of works that transmit SEM whenever an event happens. SEM is processed once and then invoked through a cue over the network. It does save resources by reducing the overhead of repeated information transmitted. By adding lightweight communication protocols, such as CoAP and MQTT, the study concludes that response time is boosted in relation to UPnP and WebSocket and are recommended for networked event-based mulsemedia systems. There were statistically significant differences in response time between those protocols. Moreover, these time references can be used for the design of mulsemedia systems with temporal restrictions. This study is available in the thesis but preliminary research was published in $[12,18]$.

\subsection{Users' QoE Using the Framework}

With the aim to compare user QoE whilst consuming mulsemedia from a monolithic system (SEMP) and a decoupled one (the proposed framework), 40 participants (20 male and 20 female) divided into 2 groups were invited to take part in this subjective experiment. Each group watched 5 different videos in a randomized order on the framework and SEMP without being aware of it. The Philips amBX Premium Kit (wind fans, vibration bar, and external lighting) was employed to render the sensory effects for both systems. The study concludes that no statistically significant difference in userperceived QoE was found between the two systems under different perspectives such as overall experience watching the videos with sensory effects, audiovisual experience and content, wind effects, vibration effects, external lighting effects. The levels of perceived quality, reality, synchronization, distraction, annoyance, and enjoyment were not remarkably affected when the system was changed. Moreover, an analysis of the interaction between system and video did not evince that there is a statistically significant change in 
user-perceived QoE when changing the videos for each system. Therefore, the framework's architecture, which allows the possibility of combining different technologies in a customizable fashion, can be leveraged in mulsemedia environments without decreasing levels of user QoE in comparison with a monolithic system. Parallel user QoE research using the framework has been made available in multiple studies [1, 4, 6-9].

\section{RESEARCH ACCOMPLISHMENTS}

\subsection{Sandwich PhD}

The candidate had the opportunity to be an Academic Visitor at Brunel University London, UK, for six months, supported by CAPES (88881.187844/2018-01). Alongside his coworkers, he was able to build software and hardware prototypes ${ }^{1}$ for mulsemedia, which was his object of study. From these developments, we could carry out many subjective experiments ${ }^{2}$ interviewing several participants at the University of West London, University of Wales Trinity Saint David, Middlesex University, and University of Kent. Researchers at those universities further became the candidate's coauthors in many relevant papers contributing to his formation.

\subsection{Publications and Research Dissemination}

- Journals (Impact Factor 2019):

- Saleme et al. [13] International Journal of Human-Computer Studies (IF 3.163)

- Saleme et al. [14] ACM Computing Surveys (IF 7.990)

- Saleme et al. [17] Journal of Universal Computer Science (IF 0.701)

- Saleme et al. [19] IEEE Multimedia (IF 4.962)

- Saleme et al. [20] Multimedia Systems (IF 1.563)

- de Amorim et al. [5] Multimedia Tools and Applications (IF 2.313)

- Comsa et al. [1] IEEE Multimedia (IF 4.962)

- Covaci et al. [3] IEEE Transactions on Multimedia (IF 6.051)

- Mesfin et al. [10] Multimedia Tools and Applications (IF 2.313)

- Mesfin et al. [9] IEEE Transactions on Multimedia (IF 6.051)

- Conferences (h5-index Google Scholar metrics):

- Saleme et al. [12] ACM MMSys'17 (h5-index=31)

- Saleme et al. [16] ACM MEDES'18 (h5-index=9)

- Saleme et al. [18] ACM MMSys'18 (h5-index=31)

- Covaci et al. [4] ACM Multimedia'19 (h5-index=58)

- Saleme et al. [15] SBC IHC'19 (h5-index=9)

In addition to journals and conferences, a summary of the candidate's thesis was published at ACM SIGMM (Special Interest Group in Multimedia) Records, Volume 11, Issue 4, December 2019 (ISSN 1947-4598) ${ }^{3}$. Furthermore, the paper [2] has been submitted to IEEE TVCG (IF 4.558). The candidate was also awarded two student travel grants to present papers during his $\mathrm{PhD}$ at ACM MMSys' $17^{4}$, in

\footnotetext{
${ }^{1}$ Mulsemedia Infrastructure summary available at https://bit.ly/32NdIw9

${ }^{2}$ QoE in Mulsemedia Systems summary at available https://bit.ly/2vBZr9]

${ }^{3}$ ACM SIGMM Records PhD summary available at https://bit.ly/39jYj8Z

${ }^{4} \mathrm{ACM}$ MMSys'17 presentation photo available at https://bit.ly/2wmJ3tE
}

Taipei, Taiwan, and at ACM Multimedia' $19^{5}$, in Nice, France. He was also a session volunteer for ACM Multimedia' $19^{6}$. Furthermore, he had his thesis nominated among the top six $2019 \mathrm{PhD}$ theses in Computer Science by the Brazilian Computer Society ${ }^{7}$.

\section{FINAL REMARKS AND IMPACT}

The thesis dealt with a hot topic: a conceptual architecture and a framework to support how the research community can leverage reusable mulsemedia systems in different contexts. The originality of the work relies on the multisensory excitation in addition to the usual multimedia dimension (image and sound). We highlight the nature of the work that requires not only software engineering skills, but also psychology background in addition to other side disciplines (statistics, signal processing, etc.). The work tackles and validates the problem throughout the stack, from the system to the user perception. It addresses a well-known and accepted problem and can be an impactful work. For instance, independent researchers [6-8] have taken advantage of our work to carry out their research using our set of tools.

The manuscript is composed of six chapters developed on 147 pages. It is supplemented with a meaningful and detailed annex to secure reproducibility of the experiments conducted along the research work. The research contributions rely on a complex and solid experimental setup. The work results are discussed around research questions along with the development of most advanced multimedia technology. The references try to represent the interdisciplinary dimension of the conducted research (advanced techniques in software engineering and systems, HCI, and psychology), which is inherent with the nature of the emerging sciences of Mulsemedia Systems and Quality of Experience.

From the thesis, the dissemination efforts are substantial with relevant direct and indirect outputs. These contributions are important for the community as they are addressing hot but poorly addressed topics. They have the potential to constitute a milestone for other researchers by fostering the area of $\mathrm{HCI}$ and mulsemedia in the sense that multisensory research can be leveraged to pave new ways and boost several application areas, such as entertainment, healthcare, education, culture, and marketing. Furthermore, the work was exposed to different supervision culture. This has not only enriched the candidate skills, but also made him find his role/place in such context successfully.

\section{ACKNOWLEDGMENTS}

Special thanks go to the Coordenação de Aperfeiçoamento de Pessoal de Nível Superior-Brasil (CAPES)-Finance Code 88881.187844/ 2018-01 (six months), the Federal Institute of Espírito Santo (IFES), the Fundação de Amparo à Pesquisa e Inovação do Espírito Santo (FAPES)-TO: 327/2019 and 479/2019, and the Association for Computing Machinery's Special Interest Group on Multimedia (ACM SIGMM), who have conceded grant awards and financial aid to support the candidate's research activities.

\footnotetext{
${ }^{5}$ ACM Multimedia'19 presentation photo available at https://bit.ly/32K84Lo ${ }^{6} \mathrm{ACM}$ Multimedia'19 session volunteer report available at https://bit.ly/2TkYKus ${ }^{7} \mathrm{CTD} / \mathrm{CSBC}$ summary available at https://sol.sbc.org.br/index.php/ctd/article/view/ 11362
} 


\section{REFERENCES}

[1] I-S Comsa, E. B. Saleme, A. Covaci, G. Mesfin, R. Trestian, C. A. S. Santos, and G. Ghinea. 2020. Do I Smell Coffee? The Tale of a $360^{\circ}$ Mulsemedia Experience. IEEE MultiMedia 27, 1 (2020), 27-36.

[2] A. Covaci, E. B. Saleme, G. Mesfin, I-S. Comsa, R. Trestian, C. A. S. Santos, and G Ghinea. 2020. Multisensory $360^{\circ}$ Videos Mediate Presence. IEEE Transactions on Visualization and Computer Graphics (2020), 1-9. Submitted.

[3] A. Covaci, E. B. Saleme, G. A. Mesfin, N. Hussain, E. Kani-Zabihi, and G. Ghinea 2020. How Do We Experience Crossmodal Correspondent Mulsemedia Content? IEEE Transactions on Multimedia 22, 5 (2020), 1249-1258.

[4] A. Covaci, R. Trestian, E. B. Saleme, I-S Comsa, G. Mesfin, C. A. S. Santos, and G. Ghinea. 2019. $360^{\circ}$ Mulsemedia: A Way to Improve Subjective QoE in $360^{\circ}$ Videos. In Proc. of the 27th ACM Int. Conf. on Multimedia (Nice, France). ACM, 2378-2386. https://doi.org/10.1145/3343031.3350954

[5] M. N. de Amorim, E. B. Saleme, F. R. de Assis Neto, C. A. S. Santos, and G. Ghinea 2019. Crowdsourcing authoring of sensory effects on videos. Multimedia Tools and Applications 78, 14 (01 Jul 2019), 19201-19227. https://doi.org/10.1007/s11042019-7312-2

[6] L. Jalal, M. Anedda, V. Popescu, and M. Murroni. 2018. QoE Assessment for Broadcasting Multi Sensorial Media in Smart Home Scenario. In 2018 IEEE International Symposium on Broadband Multimedia Systems and Broadcasting (BMSB). 1-5. https://doi.org/10.1109/BMSB.2018.8436875

[7] L. Jalal, M. Anedda, V. Popescu, and M. Murroni. 2018. QoE Assessment for IoT-Based Multi Sensorial Media Broadcasting. IEEE Trans. Broadcast. 64, 2 (June 2018), 552-560. https://doi.org/10.1109/TBC.2018.2823914

[8] L. Jalal and M. Murroni. 2019. The Impact of Multi-Sensorial Media in Smart Home Scenario on User Experience and Emotions. In 2019 IEEE International Symposium on Broadband Multimedia Systems and Broadcasting (BMSB). 1-6. https://doi.org/10.1109/BMSB47279.2019.8971922

[9] G. Mesfin, E. B. Saleme, O. Ademoye, E. Kani-Zabihi, C. A. S. Santos, and G. Ghinea. 2020. Less is (Just as Good as) More - an Investigation of Olfactory Intensity and Hedonic Valence in Mulsemedia QoE using Heart Rate and Eye Tracking. IEEE Transactions on Multimedia (2020), 11

[10] G. A. Mesfin, N. Hussain, E. Kani-Zabihi, A. Covaci, E. B. Saleme, and G. Ghinea 2020. QoE of Cross-modally Mapped Mulsemedia: an Assessment Using Eye Gaze and Heart Rate. Multimedia Tools and Applications (2020), 21.
[11] E. B. Saleme. 2019. A Conceptual Architecture and a Framework for Dealing with Variability in Mulsemedia Systems. Ph.D. Dissertation. Federal University of Espírito Santo. Online; Accessed on 03/09/2020. Available at http://informatica. ufes.br/pt-br/pos-graduacao/PPGI/detalhes-da-tese?id=13927.

[12] E. B. Saleme, J. R. Celestrini, and C. A. S. Santos. 2017. Time Evaluation for the Integration of a Gestural Interactive Application with a Distributed Mulsemedia Platform. In Proc. of the 8th ACM on Multimedia Systems Conference. ACM, 308314. https://doi.org/10.1145/3083187.3084013

[13] E. B. Saleme, A. Covaci, G. Assres, I-S. Comsa, R. Trestian, C. A.S. Santos, and G. Ghinea. 2021. The Influence of Human Factors on $360^{\circ}$ Mulsemedia QoE. International fournal of Human-Computer Studies 146 (2021), 102550. https: //doi.org/10.1016/j.ijhcs.2020.102550

[14] E. B. Saleme, A. Covaci, G. Mesfin, C. A. S. Santos, and G. Ghinea. 2019. Mulsemedia DIY: A Survey of Devices and a Tutorial for Building Your Own Mulsemedia Environment. Comput. Surveys 52, 3, Article 58 (June 2019), 29 pages.

[15] E. B. Saleme, A. Covaci, G. Mesfin, C. A. S. Santos, and G. Ghinea. 2019. Sumarização de Dispositivos de Efeitos Multissensoriais para Interações HumanoComputador. In Extended Proceedings of IHC '19: XVIII Brazilian Symposium on Human Factors in Computing Systems (IHC '19). SBC, 2.

[16] E. B. Saleme, C. A. S. Santos, R. A. Falbo, G. Ghinea, and F. Andres. 2018. Towards a Reference Ontology on Mulsemedia Systems. In Proceedings of the 10th International Conference on Management of Digital EcoSystems (Tokyo, Japan) (MEDES '18). ACM, 23-30. https://doi.org/10.1145/3281375.3281378

[17] E. B. Saleme, C. A. S. Santos, R. A. Falbo, G. Ghinea, and F. Andres. 2019. MulseOnto: a Reference Ontology to Support the Design of Mulsemedia Systems. Journal of Universal Computer Science 25, 13 (2019), 26.

[18] E. B. Saleme, C. A. S. Santos, and G. Ghinea. 2018. Improving Response Time Interval in Networked Event-based Mulsemedia Systems. In Proceedings of the 9th ACM Multimedia Systems Conference (Amsterdam, Netherlands) (MMSys '18). ACM, 216-224. https://doi.org/10.1145/3204949.3204965

[19] E. B. Saleme, C. A. S. Santos, and G. Ghinea. 2019. Coping With the Challenges of Delivering Multiple Sensorial Media. IEEE MultiMedia 26, 2 (April 2019), 66-75. https://doi.org/10.1109/MMUL.2018.2873565

[20] E. B. Saleme, C. A. S. Santos, and G. Ghinea. 2019. A mulsemedia framework for delivering sensory effects to heterogeneous systems. Multimedia Systems 25, 4 (01 Aug 2019), 421-447. https://doi.org/10.1007/s00530-019-00618-8 